OCT 12 Pि?

UCRL-7762 Rev. I

\author{
University of California \\ Ernest 0 . Lawrence \\ Radiation Laboratory
}

CALCULATION OF THE SHOCK WAVE FROM AN
UNDERGROUND NUCLEAR EXPLOSION IN GRANITE

Livermore, California 


\section{DISCLAIMER}

This report was prepared as an account of work sponsored by an agency of the United States Government. Neither the United States Government nor any agency Thereof, nor any of their employees, makes any warranty, express or implied, or assumes any legal liability or responsibility for the accuracy, completeness, or usefulness of any information, apparatus, product, or process disclosed, or represents that its use would not infringe privately owned rights. Reference herein to any specific commercial product, process, or service by trade name, trademark, manufacturer, or otherwise does not necessarily constitute or imply its endorsement, recommendation, or favoring by the United States Government or any agency thereof. The views and opinions of authors expressed herein do not necessarily state or reflect those of the United States Government or any agency thereof. 


\section{DISCLAIMER}

Portions of this document may be illegible in electronic image products. Images are produced from the best available original document. 


\author{
UNIVERSITY OF CALIFORNIA \\ Lawrence Radiation Laboratory \\ Livermore, California \\ Contract No. W-7405-eng-48
}

CALCULATION OF THE SHOCK WAVE FROM AN UNDERGROUND NUCLEAR EXPLOSION IN GRANITE

Theodore R. Butkovich

May 7, 1964

(Presented at the Third Plowshare Symposium,

"Engineer.ing with Nuclear Explosives," April 21-23, 1964) 


\title{
CALCULATION OF THE SHOCK WAVE FROM AN UNDERGROUND NUCLEAR EXPLOSION IN GRANITE
}

\author{
Theodore R. Butkovich \\ Lawrence Radiation Laboratory, University of California \\ Livermore, California \\ May 7, 1964
}

\begin{abstract}
The capability of calculating the close-in effects of the shock wave from an underground nuclear explosion has been demonstrated. Agreement was obtained between calculation and measurements using a spherically symmetric, hydrodynamic, elastic-plastic code called SOC for the Hardhat event, a 5-kiloton nuclear detonation in granite. This capability is dependent upon having a more or less complete description of the elastic and dynamic properties of the materials involved. When this information is available, agreement within the limits of uncertainty of the measurements can be calculated for peak pressures, peak particle velocities, shock wave time of arrival, and pressure pulse shapes.
\end{abstract}

\section{INTRODUCTION}

The capability of predicting the phenomena from underground nuclear explosions on the surrounding media is becoming increasingly important. These predictions are useful in a number of ways. Certain engineering criteria regarding such things as stemming and placement of surface installations. can be established. Possible damage to existing underground structures from shock effects can be determined. Shock wave propagation is also important in crater formation from buried charges.

In any underground nuclear explosion, the shock front that propagates from the shot point carries with it energy from the explosion, and distributes this energy by doing work on the surrounding material. In the process, the material undergoes changes in both its physical and mechanical states. If enough energy is deposited in the material, it will vaporize or melt thus changing its physical state, or cause it to crush or crack. 
During the past few years, special computer codes have been developed for predicting the close-in phenomena of underground nuclear explosions using the laws of physics, and the knowledge of the properties of the materials in which the detonations occur. As a consequence, a better understanding of experimental observations and measurements has evolved.

A spherically symmetric, Lagrangian, hydrodynamic-elastic-plastic code called UNEC (Underground Nuclear Explosion Code)(Nuckolls, 1959), was used in earlier calculations. Presently, a new code called SOC (Seidl, 1964) is being used in making these calculations. SOC is similar to UNEC in that it makes a rather direct use of an experimentally determined shock Hugoniot, but differs in that it uses different equations for calculating elastic-plastic behavior and internal energy. SOC also allows for strainrate effects such as occur during pressure buildup and decay at the wave front.

Calculations, using the SOC code, were made for the Hardhat event, a 5-kiloton nuclear explosion. The device was detonated at the bottom of a 950-foot-deep, vertical hole in granite at the Nevada Test Site. The Hardhat event was chosen for the se calculations, because of the rather large number of different close-in measurements that were made in a range extending from the hydrodynamic to the elastic regions.

\section{MEASÜREMENTS}

For the Hardhat event, a variety of close-in measurements were made on a horizontal radius from the detonation point. An access shaft and tunnel had been provided, and holes were drilled from the tunnel for instrumentation (Fig. 1).

In the hydrodynamic region, times of arrival of the shock wave were measured with special transducers in the range from 7.35 to 24.1 meters (24 to $79 \mathrm{ft}$ ) (Chabai and Bass, 1963). Peak pressure measurements were made in two locations, one of 460 kilobars at 5.51 meters ( $18.1 \mathrm{ft}$ )(Chabai and Bass; 1963), and another of 664 kilobars at 4.85 meters $(15.9 \mathrm{ft})$ (Lombard, 1963).

In the elastic region, there was an array of accelerometers, velocity and displacement gauges, and stress and strain measurement instruments distributed in the range from 78 to 460 meters (256 to $1500 \mathrm{ft}$ )(Perret, 1963, and Swift, 1962). From this, some measurements 
- sandia corP. INSTRUMENTATION

- LRL INSTRUMENTATION

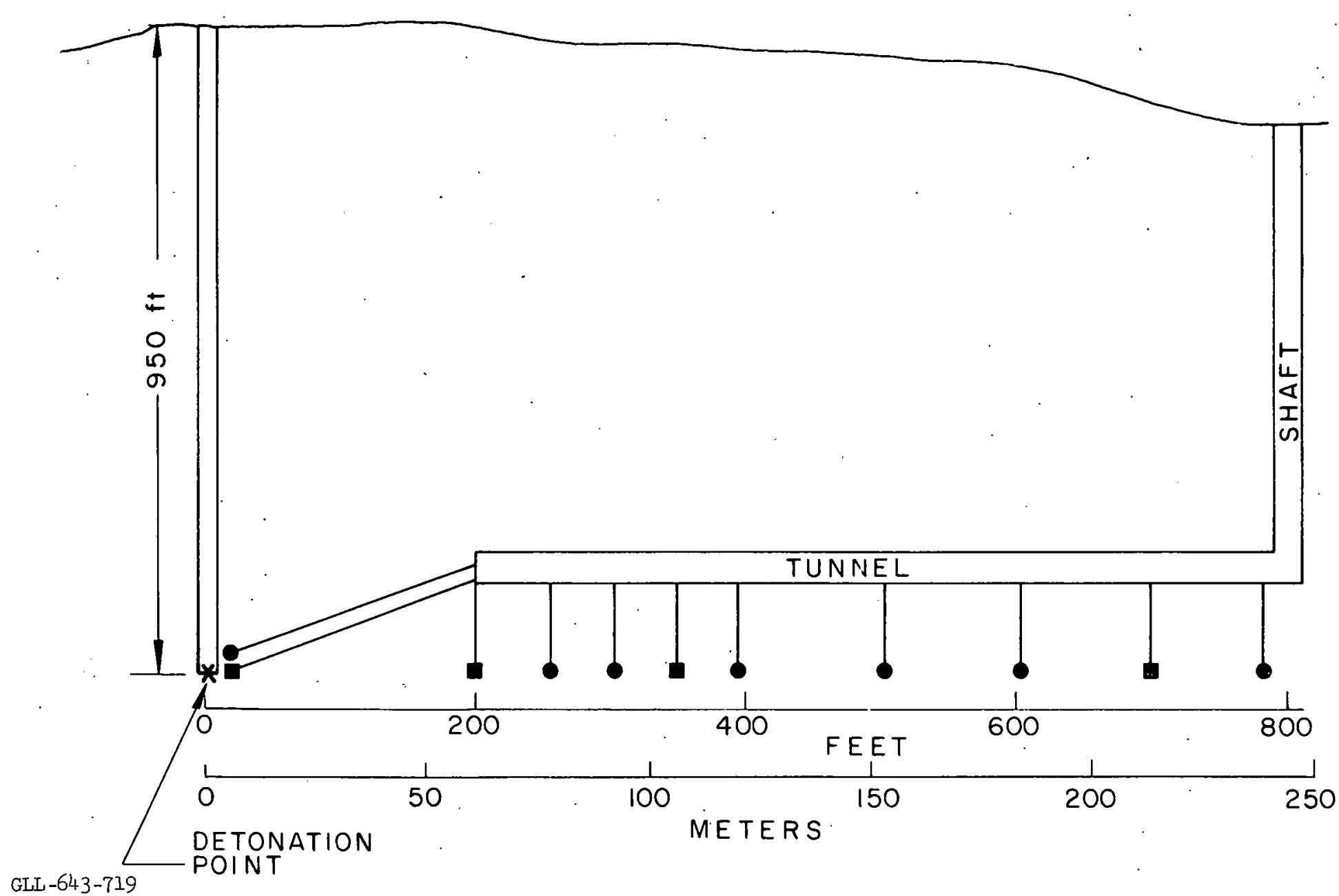

Fig. 1. Layout of instrumentation of Hardhat event. 
are of particular interest here. The time of arrival as determined by the accelerometers are shown in Fig. 2. The average velocity of the shock wave was 5526 meters $/$ second $(18,130 \mathrm{ft} / \mathrm{sec})$. With this shock velocity $U_{s}$ and the peak velocity measurements $U_{p}$, peak pressures were determined from the Hugoniot relationship

$$
P=U_{p} U_{s} \rho_{0}
$$

where $\rho_{n}$ is the initial density of the material. Pressure history measurements were made in two locations (Heusinkveld et al., 1962) with peak radial stresses of 4.0 kilobars at 61 meters $(200 \mathrm{ft})$ and 1.2 kilobars at 106.7 meters $(350 \mathrm{ft})$.

\section{TIIE SOC CODE}

Before any meaningful calculation can be attempted, a more or less complete description of the materials involved has to be obtained. The Hugoniot equation of state, the shock energy to vaporize and melt, plastic yield conditions, dynamic strength properties, and elastic properties are all input to a SOC code calculation. Some of these parameters can be determined by rather well developed techniques, but others are not easily determinable and must be estimated on the basis of other related measurements.

Except in the vaporized region, the equation of state of the material is made up from experimental data. When the material is vaporized, the equation of state is expressed as a theoretical pressure-energy-density relationship normalized to the Hugoniot, and extending to the ThomasFermi-Dirac region at high energies. In the liquid and plastic states, the material is represented by the Hugoniot curve relating pressure and specific volume at the shock front. At the shock front discontinuity, a nonlinear Richtmyer-von Neumann artificial viscosity (q) is used. The wave front is determined from a maximum in $q$, which.lies at the center of the discontinuity and travels with the wave front velocity. During the unloading, the Hugoniot can be corrected to approximate the unloading isentrope, by using an appropriate Gruneisen $\Gamma$. When shock pressures are great enough to vaporize the material on unloading, a transition to the gas equation of state, is made irreversibly. 


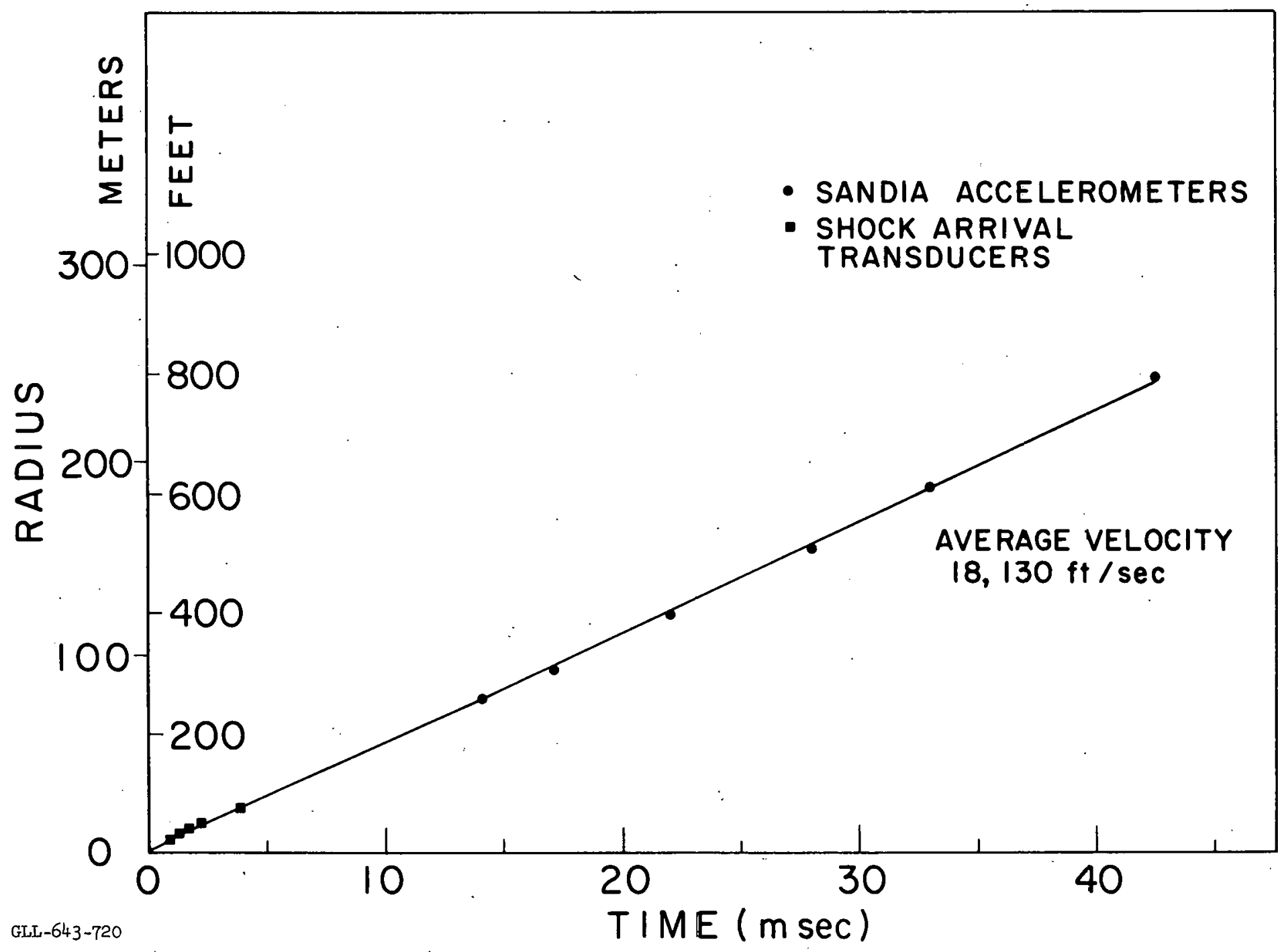

Fig. 2. Time of arrival data. 
In spherical symmetry, there are two principal stresses, $\left(\sigma_{\mathbf{r}}\right)$ normal and $\left(\sigma_{\mathrm{T}}\right)$ tangent to the wave front. That is, a distinction is made between the se and the fluid-like pressure (P), where

$$
P=\frac{\sigma_{r}+2 \sigma_{T}}{3}
$$

In the liquid state, the material is isotropic and the shear is zero. However,

$$
\sigma_{r}=P+\frac{4}{3} K \text {, and } \sigma_{T}=P-\frac{2}{3} K
$$

where $K$, the so-called stress deviator is expressed by

$$
K=\frac{\sigma_{\dddot{r}}-\sigma_{T} T}{2}
$$

$\mathrm{K}$. is calculated differently if the material is crushed.

The plastic yield conditions are expressed in terms of K (Seidl, 1964), where $K$ is equal to or less than the yield stress. For many materials, the yield stress is a function of the strain rate. When knowledge of this behavior is available, different yield conditions can be imposed according to whether the strain rates are high, as occurs at the shock front, or the pressure is slowly rising, or falling off on unloading.

The elastic region of the pressure-density curve is required to agree with sonic velocities in the material. In an isotropic elastic medium, the two characteristic sonic velocities, longitudinal $\left(v_{\ell}\right)$ and shear $\left(v_{s}\right)$, are related by

$$
v_{\ell}^{2} P_{0}=k+\frac{4}{3} G
$$

and

$$
v_{s}^{2} \rho_{0}=G
$$

where $G$ is the shear modulus. $\rho_{0}, v_{\ell}$, and $v_{s}$ are taken from in-situ measurements. The bulk modulus (k) can also be obtained from hydrostatic measurements. Stephens (1963) has shown that excellent agreement occurs between hydrostatic and dynamic measurements in the elastic region for eight different rock types. 
Dynamic strength properties of the rock are less easily determinable. The bulk tensile strength of most rock masses are zero, or at most very small, because of the highly fractured state in which they are usually found. Dynamic compressive strength of rocks are not as easily obtained, and in most cases must be estimated, perhaps, something like twice or three times static measurements. In the case where the material has open cracks, the compressive stress that can be supported without crushing the material is always less than when cracks are closed and also depend on the strain rate. If the material does crush, then it is assumed that a type of Coulomb friction exists, somewhat like the resistance to shear for loose sand.

At the start of a calculation, the material is divided into two or more regions, a central gas region into which the energy from the explosion is put as internal energy of the gas, and the regions outside in which the material. is initially plastic-elarstic. The regions are divided.into equal thickness zones to the outside. This is the ground surface in a vertical calculation, or extends somewhat beyond the region of interest in the horizontal case. After the shock wave has passed and the energy from the explosion distributed, the material state of each zone may have changed by expanding the vaporized region or melting it, depending on whether the internal energy to vaporize or melt was exceeded. Crushed or cracked regions form if the peak stresses that developed in each region exceed the crushing or tensile strength, respectively.

\section{THE CALCULATIONS}

For the Hardhat event, predictions were made based on a 5 -kiloton nuclear explosion, 950 feet below the surface in granite (Seidl, 1.962) using the best available data at that time. These predictions were useful in determining instrument placement, and for range and time' settings of the measuring equipment. Since then, more data on the properties of granite have become available, and with the measurements to compare with results; adjustments of some of the input parameters for granite used in the original calculation were made to cause better agreement.

In determining the dynamic equation of state of a material, measure:ments are made in the laboratory by subjecting representative samples of the material to strong shocks generated by high explosives. Lombard (1961) 
has compiled data of shock velocity $\left(U_{s}\right)$ and particle velocity ( $\left.U_{p}\right)$ on a number of rock types, amongst which is granite. From the se measurements the so-called Rankine-Hugoniot conditions can be obtained:

$$
\begin{aligned}
& P-P_{0}=\rho_{0} U_{s} U_{p} \\
& \rho / \rho_{0}-1=\frac{U_{p}}{U_{s}-U_{p}}, \\
& E-E_{0}=\frac{P-P_{0}}{2}\left(1 / \rho_{0}-1 / \rho\right)
\end{aligned}
$$

where $P$ is pressure, E specific internal energy, and $\rho$ the instantaneous density. The subscripts refer to initial values. Figure.3 is a plot of the data for granite. The scatter at the lower pressures is due to several causes. An elastic precursor of about 40 kilobars has been measured for granite (Grine, 1960). This means that a two-wave structure exists to about 320 kilobars; above which the shock velocity is greater than the dilatational sonic velocity. A number of polymorphic transitions of the mineral constituents of granite below 320 kilobars further complicate the interpretation of the measurements.

In the elastic region, the equation of statc of granite is defined by the bulk modulus and the shear modulus. Figure 4 is a plot of the granite Hugoniot, which is put into the SOC code as a linearly interpolated $P$ vs $\mu$ table, where $\mu=\rho / \rho_{0}-1$. In-situ seismic measurements of the dilatational and shear velocities in granite are 5440 meters $/ \mathrm{sec}(17,850 \mathrm{ft} / \mathrm{sec})$ and $3050 \mathrm{~meters} / \mathrm{sec}(10,000 \mathrm{ft} / \mathrm{sec}$ ), respectively (United Electrodynamics, 1962). The average measured wave front velocity of 5526 meters/sec $(18,310 \mathrm{ft} / \mathrm{sec})$, is in good agreement with the in-situ seismic velocities. Derived from the seismic measurements, the bulk modulus and shear modulus used in the calculations were 0.361 and 0.315 megabar, respectively。 This corresponds to a wave front velocity of 5380 meters $/ \mathrm{sec}(17,646 \mathrm{ft} / \mathrm{sec})$ in the elastic region. Since the wave front velocity is greater at pressures above about 320 kilobars, the average velocity is somewhat higher, so as to give good agreement with the shock wave time of arrival measurements.

The dynamic properties of granite were estimated and adjusted to obtain good agreement between measurement and calculation. To do this a 


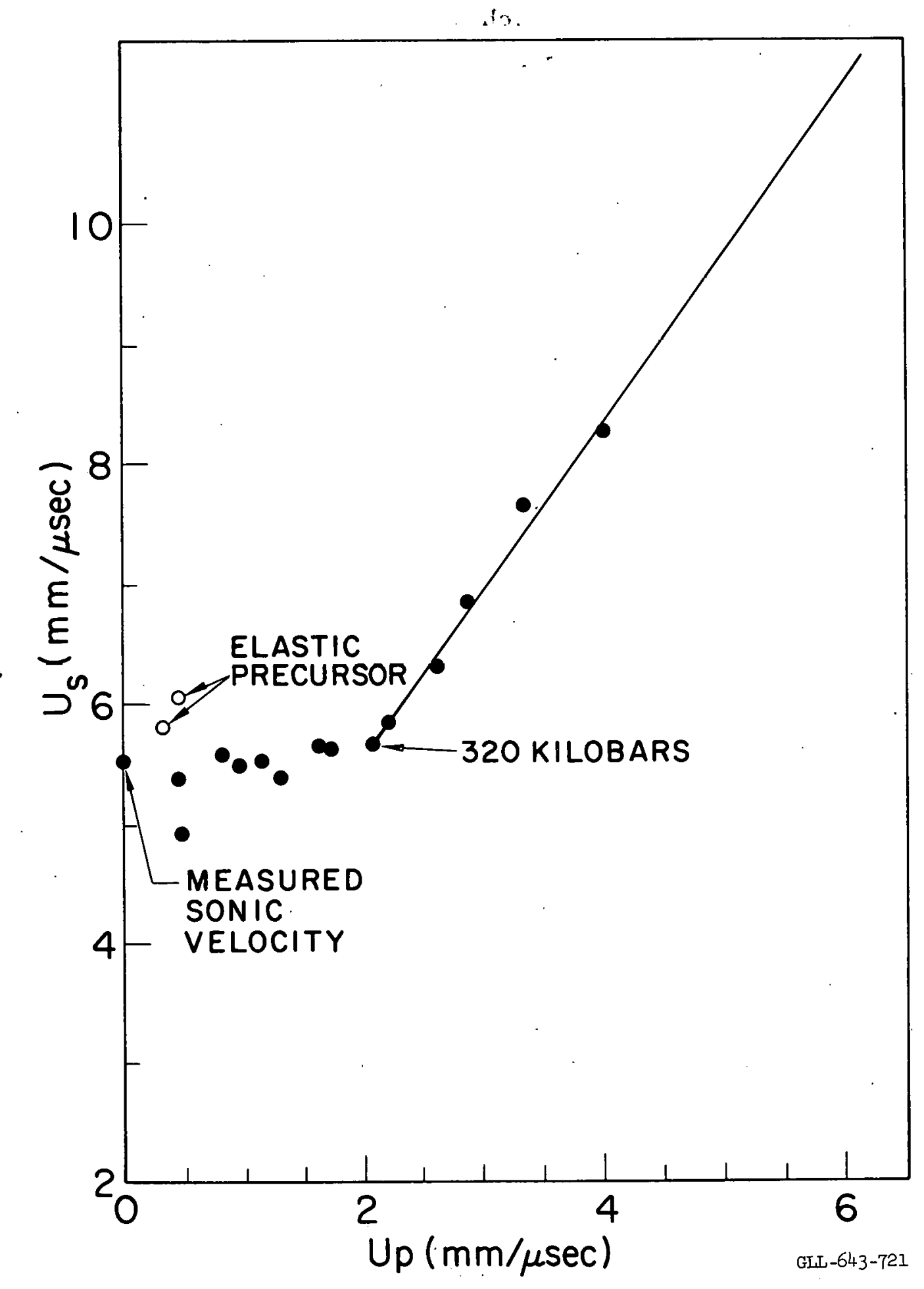

Fig. 3. Hugoniot measurements for granite. 


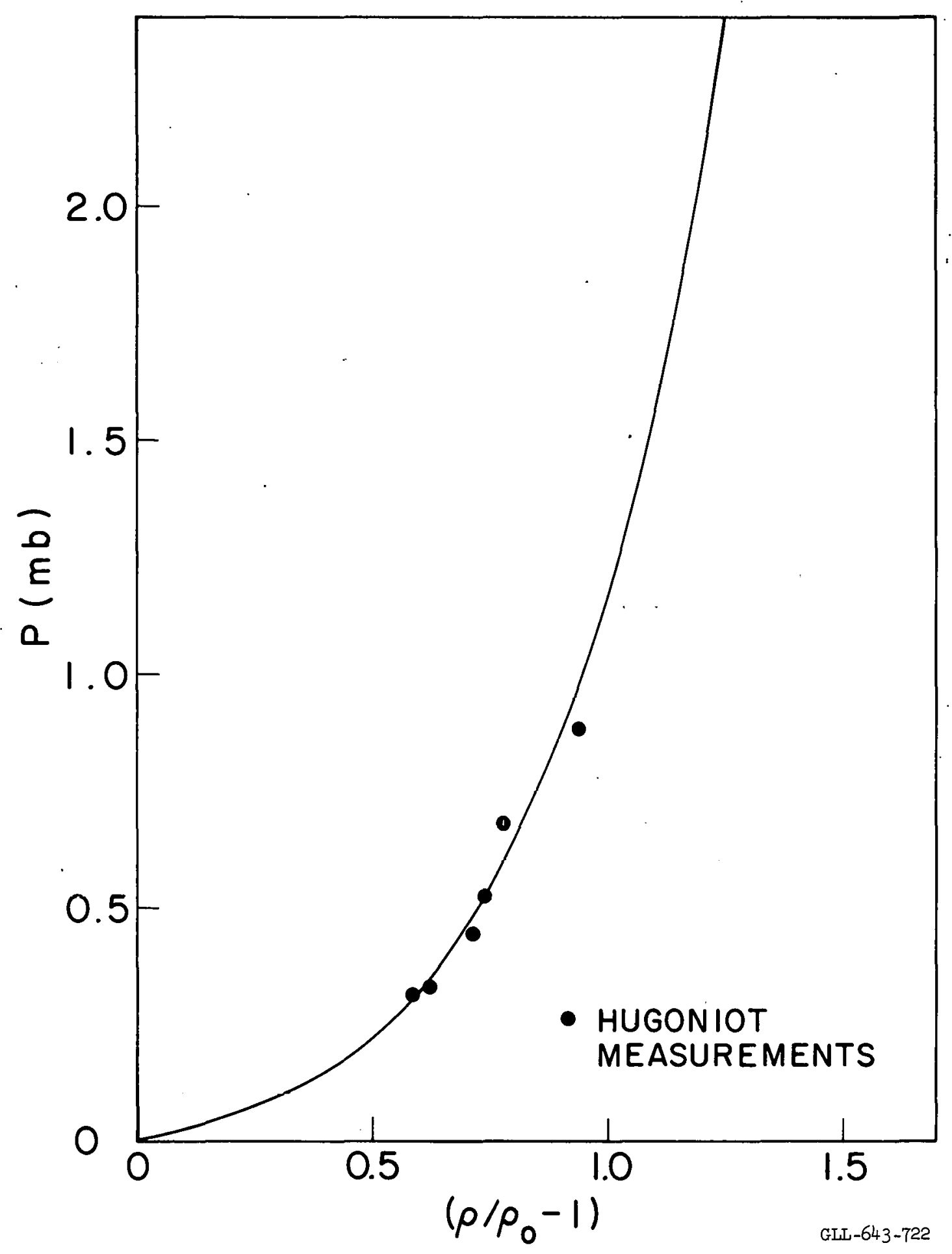

Fig. 4. Shock Hugoniot for granite. 
number of parameter studies were carried out by varying specific input values to the code.

The dynamic yield stress for high strain rates or fast-rising pressure pulses was made to be consistent with the measured 40-kilobar elastic precursor. However, the yield stress for a slowly rising pressure pulse was varied from 10 to 1 kilobars. Figure 5 shows the results for calculations. using 10, 5, and 2.5 kilobars. Reducing the yield stress further to 1 kilobar caused no further change within the limits of the calculation. These results suggest that when the material deforms plastically at a lower yield stress, more energy is deposited in the plastic state leaving less energy available for the farther out regions; hence, the peak pressures drop off more rapidly.

Birch (1942) reports static measurements of compressive strength for granite of 10 kilobars for confined tests and 1.5 kilobars for unconfined tests. The dynamic compressive strengths were assumed to be twice the values from static tests; 20 kilobars for the case when the material is confined with no open cracks and high strain rates, and 3 kilobars for the unconfined case with open cracks or for slowly rising pressure pulses. A parameter study varying the compressive strength for the unconfined case was made to determine the effect on stress pulse shape. Figure 6 shows.the results of this study where the calculated radial and tangential stress pulses are plotted for the zone nearest 61.8 meters $(203 \mathrm{ft})$ from the detonation center, where stress history measurements were made. For the compressive strength of 10 and 5 kilobars, the material cracks when the tangential stress goes to zero. The material remains elastic-plastic and the radial stress remains high. For a compressive strength of 3 kilobars, the material crushes when the peak stress exceeds this value, and the radial stress pulse shape very nearly approximates the measured pulse shape. The higher values of compressive strength make the material more rigid than it is in reality. It is of interest to note that the Hardhat tunnel collapsed completely out to a radius of 137 meters (450 ft) (Lombard and Cauthen, 1964). The peak radial stress at this point is 1.2 kilobars. Total tunnel collapse should occur somewhat beyond the limit of crushing, as the failure was primarily due to spall.

The bulk tensile strength used in all these calculations was zero; however, a calculation was made assuming the tensile strength equal to the overburden of 77 bars. No appreciable differences were noted in the stress pulse shapes. 


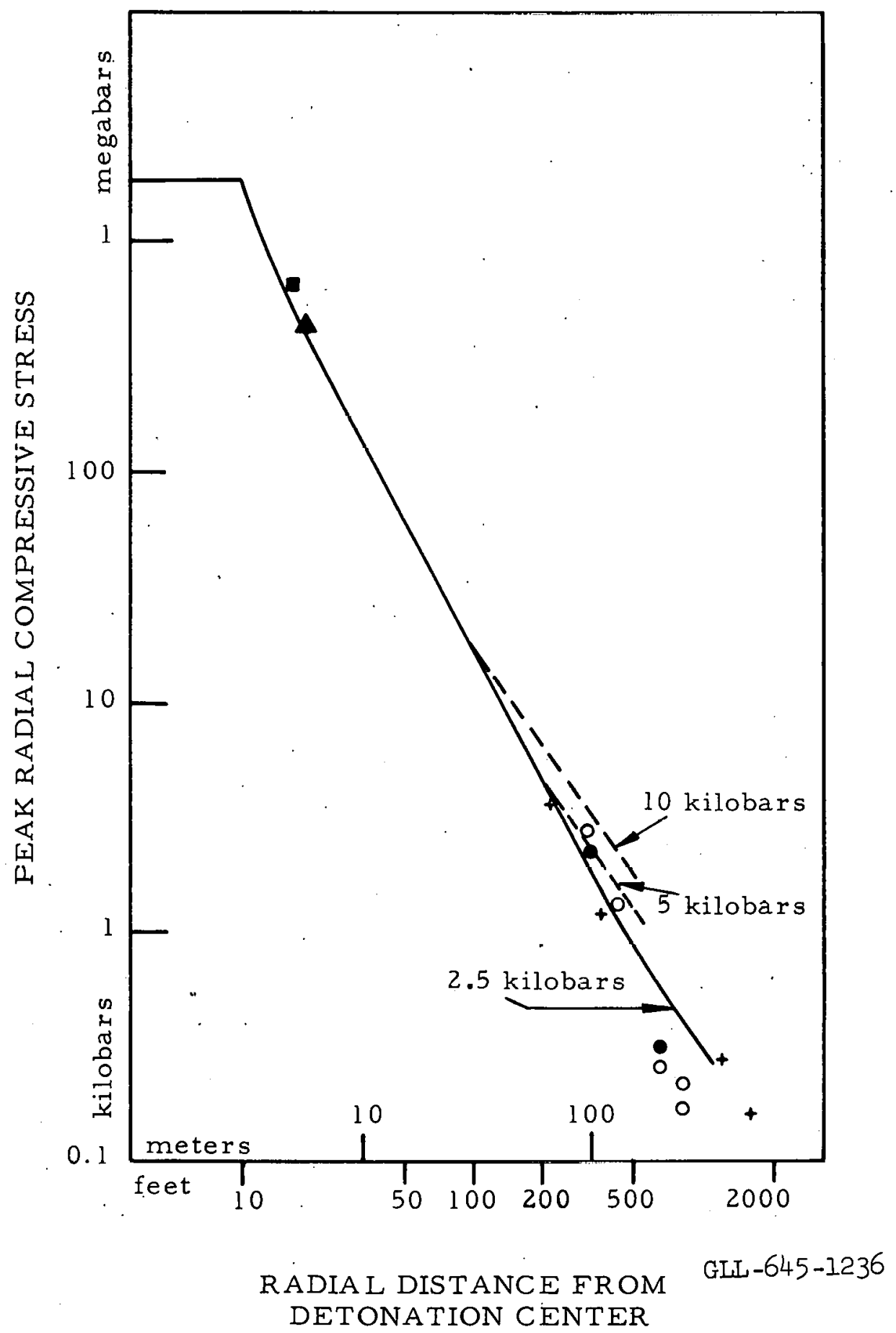

Fig. 5. Results of parameter study varying yield stress for a slowly rising pressure pulse. 


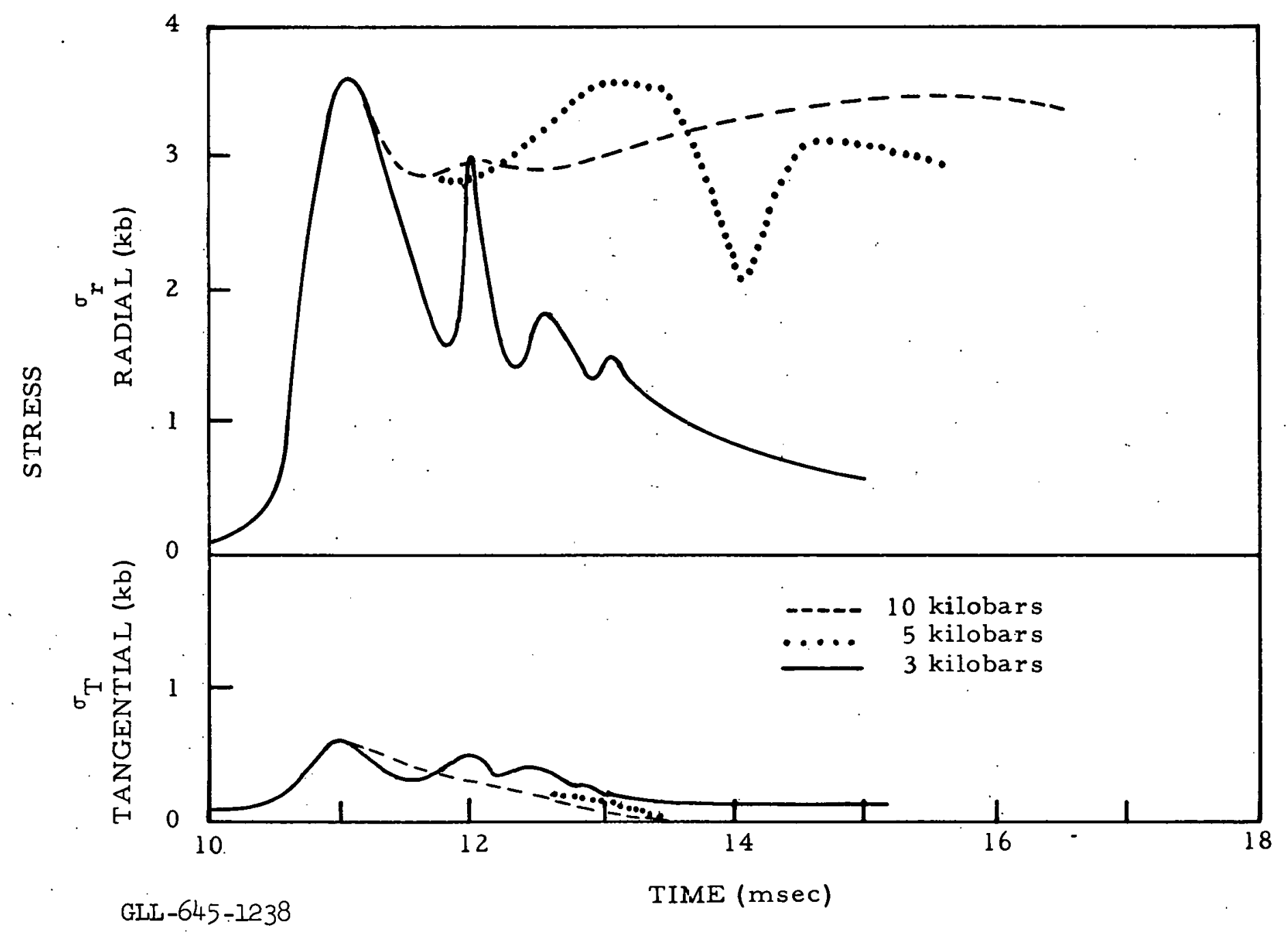

Fig. 6. Results of parameter study varying the compressive strength for the unconfined case. 
The Gruneisen $\Gamma$ was set equal to 1.0, knowing that it was greater than zero and less than two. Since the calculation was concerned primarily with material behavior. near the shock front, a small error in the unloading isentrope would have little effect there. Vaporization occurred behind the shock front when the internal energy exceeded $0.584 \times 10^{12}$ ergs per original cc. Melting occurred when the internal energy was greater than $0.093 \times 10^{12}$ ergs per original cc. These values are equivalent to a shock vaporization pressure of 2.14 megabars and a shock melting pressure of 456 kilobars, if the Gruneisen $\Gamma$ was zero, and the Hugoniot becomes the unloading isentrope. With $\Gamma$ being set equal to one, the se pressures are slightly higher.

The calculation was made in two steps. First, a fine-zoned case was run to more precisely determine the fall-off of peak pressure with distance in the region above 100 kilobars, and the limit of vaporization. The 5 kilotons were distributed uniformly as internal energy of a sphere of iron gas, with an average density and volume equal to that of the device canister: The second case used coarser zoning to cover the region below 100 kilobars, where the pressure was falling off less rapidly. It assumed that the initial density of the gas was approximately equal to the total mass of material vaporized, divided by the volume of the vaporized region. In both cases, the average initial bulk density of granite was $2.67 \mathrm{~g} / \mathrm{cm}^{3}$.

The peak shock pressure as a function of distance from the detonation center is shown in Fig. 7, along with limits of vaporization, melting, crushing and cracking. Peak pressure falls off as $\mathrm{r}^{-1.94}$ in the region below approximately 1 megabar for a 5-kiloton detonation in granite.

Pressure-history measurements (Heusinkveld et al., 1962) at 61.8 meters (203 ft) and 109.7 meters (360 ft) are plotted in Fig. 8. Superimposed on these plots are the calculated pressure histories for the nearest zone position. From this, the curves are displaced $0.03 \mathrm{msec}$ in time at most. The oscillatory shape at the calculated curves is due to the mathematical method the code uses. The true pulse shape should be more like the envelope formed by the peaks. The large discrepancy in arrival time between the calculated and observed pressure pulse at $360 \mathrm{ft}$ is attributed to an error in instrument position determination, since this is the only one of many measurements that does not fall on the shock time of arrival curve. 


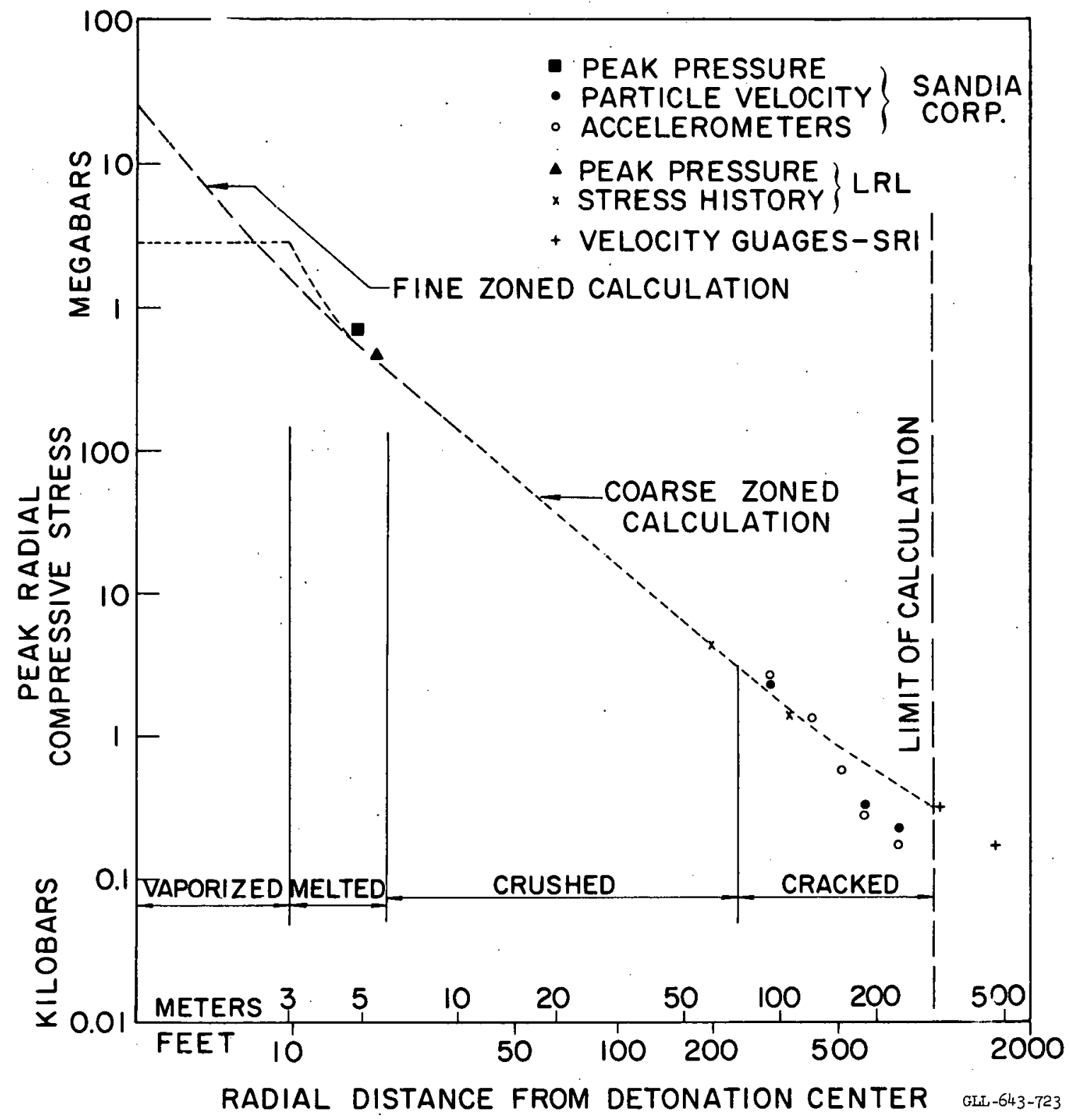

Fig. 7. Hardhat event, peak radial stress vs radius. 


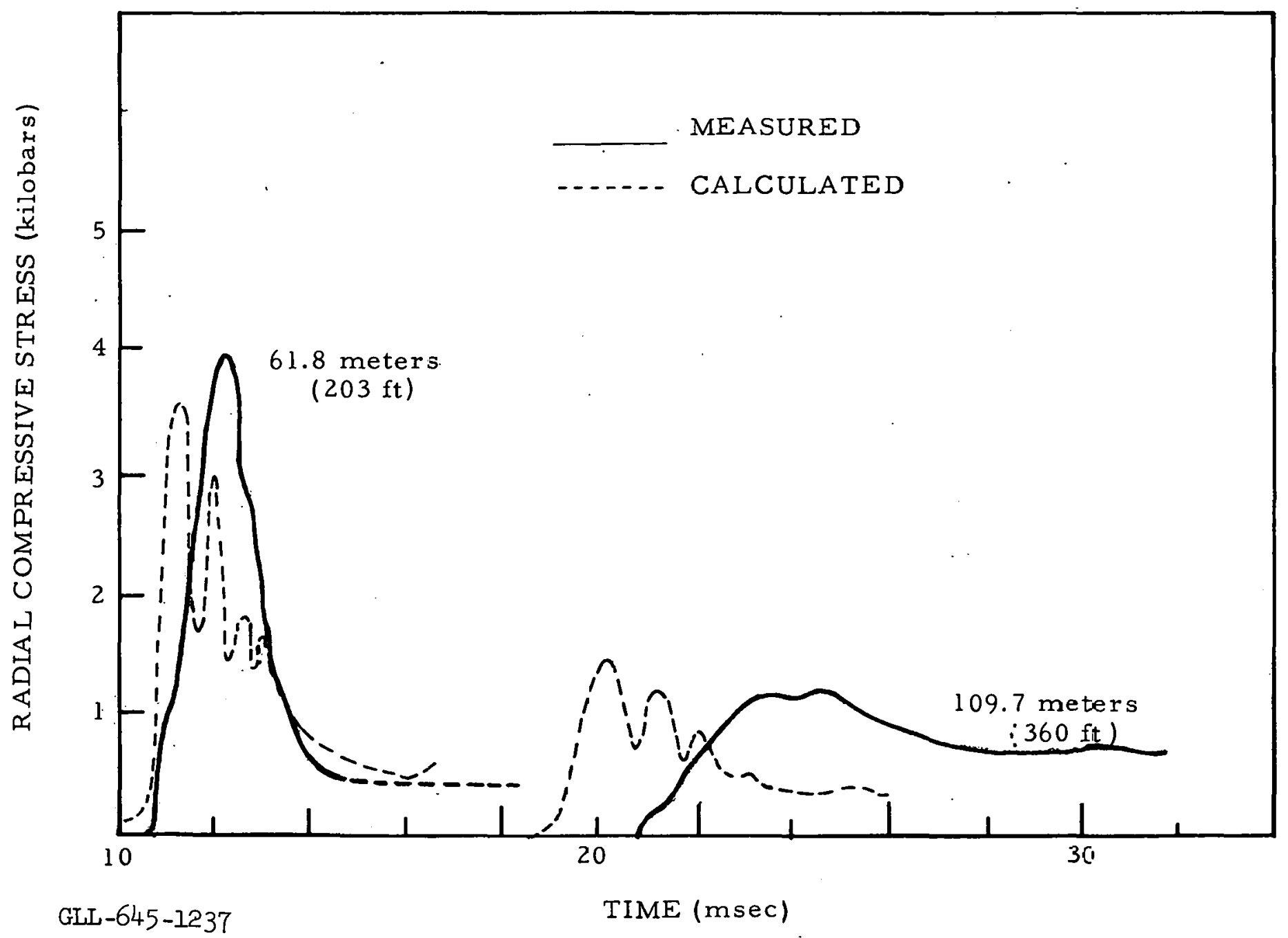

Fig- 8. Pressure-history measurements. 
The peak particle velocity vs radius is plotted in Fig. 9. The measurements shown were obtained by integration of the acceleration-time data, and by direct observation of velocity gauge signals. Sandia data seem to indicate the velocity to be falling off faster than that calculated. However, measurements by Swift (1962) at 457 meters (1500 ft) seem to agree better with the calculation.

\section{DISCUSSION OF RESULTS}

The agreement between calculation and measurements for the Hardhat event has demonstrated the capability for predicting with considerable accuracy the close-in effects of the shock wave from an underground nuclear explosion. The differences that are noted are due to uncertainties in the measurements of the phenomena, in measurements of the material parameters, and the fact that a spherical model was assumed.

Measurements of material properties upon which the input parameters are based are made on selected samples or in areas that are similar to the detonation region, but are not necessarily representative. Often there are large variations in the structural geology for a given type of material within one area. The properties of the materials upon which the calculation is based must exhibit an average behavior of the medium.

The code uses a spherical model, whereas in reality the geometry of the device room in an underground nuclear explosion is rarely spherical. Also, the detonation is not truly a point source, and a small displacement of the detonation center can mean a rather large discrepancy in peak pressure within the first few meters.

The device yield itself is based on measurements, and each measurement has an uncertainty. The uncertainty can be due to a number of sources, such as time and position resolution or instrument design and calibration. Some measurements are obviously in error and are discarded because of disagreement with other reliable values.

In the Plowshare group, a major effort is being made to better understand the phenomenology of underground nuclear detonations by code development, obtaining better input parameters to these codes, and improved measurement techniques. 


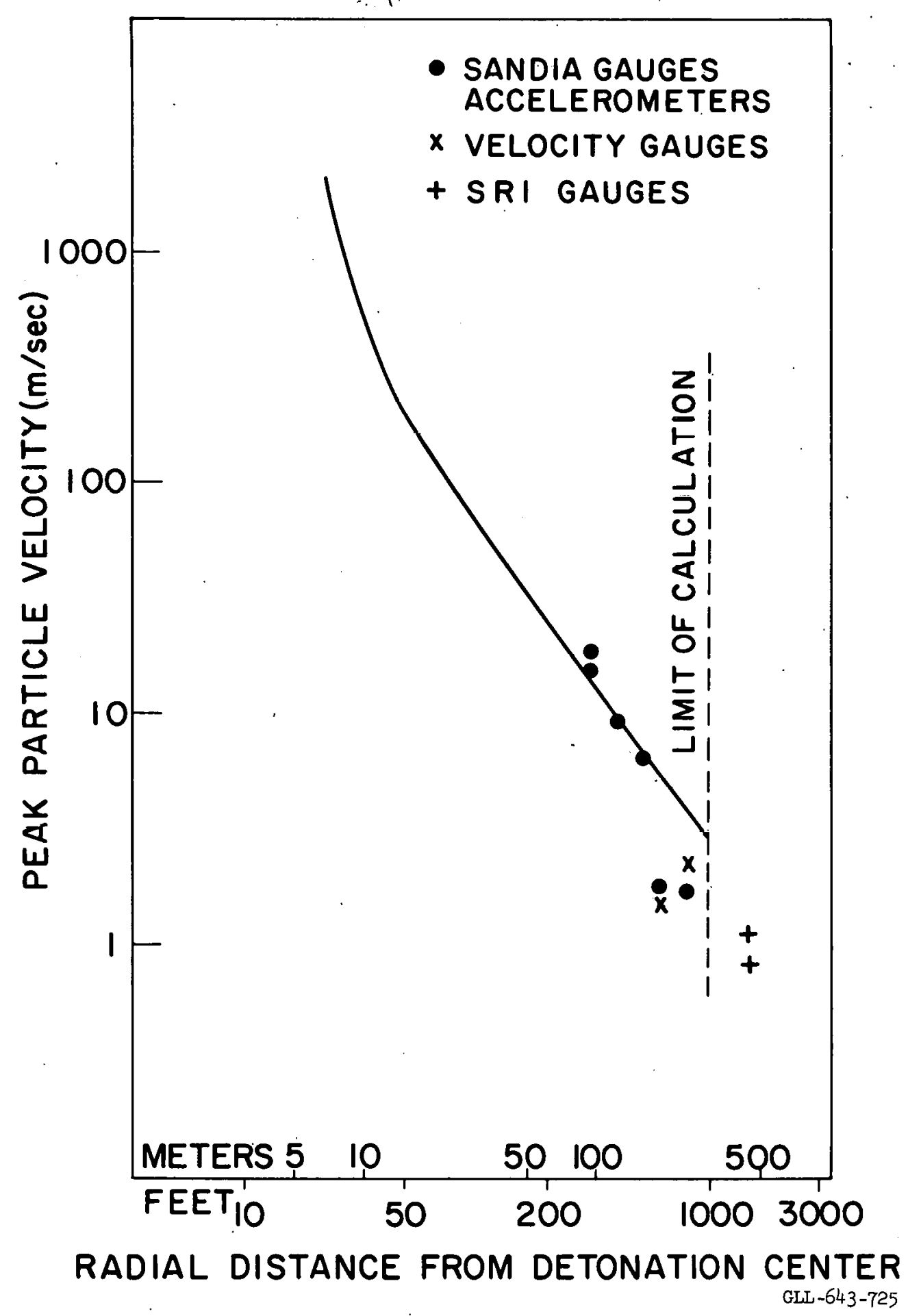

Fig. 9. Peak particle velocity vs radius. 


\section{BIB LIOGR APHY}

1. Birch, F., Handbook of Physical Constants, Geological Society of America, Special Papers 36, 1942.

2. Chabai, A.J. and Bass, R. C., Sandia Corp. Research Report, SC-4741, 1963.

3. Grine, D. R., Equation of State of Granite and Salt, Stanford Research Institute, Final Report, Project PGD-3244, 1961.

4. Heusinkveld, M., Holzer, F., Marks, R., and Harris, D., Stress History Measurements with' Piènelertrir Crystals, Hardhat Preliminary Report, Project 26.21, K Div., LRL, 1962.

5. Lombard, D. B., The Hugoniot Equation of State of Rocks, UCRL$5675,1959$.

6. Lombard, D. B., and Cauthen, L., Shock and Seismic Effects of Underground Explosions, to be published, 1964.

7. Perret, W. R., Free-Field Ground Motion Studies in Granite, Sandia Corp., POR-1803, 1961.

8. Seidl, F. G. P., Publication describing SOC code, to be published, 1964.

9. Seidl, F. G. P., Machine Calculation (Viz., SOC) of the Fall-off with Distance of the Peak Pressure and Peak Particle Velocity Produced by a $5 \mathrm{kt}$ Nuclear Explosion, 950 feet Below the Surface of a SemiInfinite Granite Medium, UCRL Internal Memorandum UOPKA 62-6, 1962 .

10. Swift, L. M., Measurement of Close-in.Earth Motion, Preliminary Report Hardhat Event, VUP-2101, 1962.

11. United Electrodynamics, Compression and Shęar Wave Velocities, Preliminary Report VUP-2500, 1962. 
This report was prepared as an account of Government sponsored work. Neither the United States, nor the Commission, nor any person acting on behalf of the Commission:

A. Makes any warranty or representation, expressed or implied, with respect to the accuracy, completeness, or usefulness of the information contained in this report, or that the use of any information, apparatus, method, or process disclosed in this report may not infringe privately owned rights; or

B. Assumes any liabilities with respect to the use of, or for damages resulting from the use of any information, apparatus, method or process disclosed in this report.

As used in the above, "person acting on behalf of the Commission" includes any employee or contractor of the commission, or employee of such contractor, to the extent that such employee or contractor of the Commission, or employee of such contractor prepares, disseminates, or provides access to, any information pursuant to his employment or contract with the Commission, or his employment with such coritractor. 